\title{
DETRENDED FLUCTUATION ANALYSES OF SHORT- TERM HEART RATE VARIABILITY IN SURGICAL INTENSIVE CARE UNITS
}

\author{
Rong-Guan Yeh ${ }^{1}$, Jiann-Shing Shieh ${ }^{1}$, Yin-Yi Han ${ }^{2}$, Yu-JungWang ${ }^{1}$, Shih-Chun Tseng ${ }^{1}$ \\ ${ }^{1}$ Department of Mechanical Engineering, Yuan Ze University, Taoyuan, \\ ${ }^{2}$ Department of Trauma, Division of Surgical Intensive Care, College of Medicine, National \\ Taiwan University, Taipei, Taiwan
}

\begin{abstract}
We examine the dynamics of complex physiologic fluctuations using methods developed very recently in statistical physics. The method based on detrended fluctuation analysis (DFA) has been used to investigate the profile of different types of physiologic states under long term (i.e., $24 \mathrm{hr}$ ) analysis of heart rate variability (HRV). In this paper, this method to investigate the output of central physiologic control system under short term (i.e., $1 \mathrm{hr}$ ) of HRV in surgical intensive care units (SICU). Electrocardiograph (ECG) signals lasting around $1 \mathrm{hr}$ were collected from ten college student volunteers as group A. Ten computes-generates white noise signals as group B also provided ECG signals lasting around $1 \mathrm{hr}$. Finally, seventeen patients representing 37 cases undergoing different types of neurosurgery were studied as group C. From this group, 25 cases were selected from 15 patients with brain injury and 12 cases were selected from 2 patients with septicemia. Group $A$ and $B$ were used as high and low limits of baseline for comparison with pathologic states in the SICU. The $\alpha$ values of DFA of groups $A, B$, and $C$ were $0.958 \pm 0.034,0.521 \pm 0.010$, and $0.815 \pm$ 0.183 , respectively. It was found that the $\alpha$ value of patients in the SICU was significantly lower $(P$ $<0.05)$ than that of healthy volunteers and significantly higher $(P<0.05)$ than white noise signals. Hence, it can be concluded that a values based on the DFA statistical concept can clearly distinguish pathologic states in SICU patients from the healthy group and from white noise signals.
\end{abstract}

Biomed Eng Appl Basis Comm, 2006(April); 18: 67-72.

Keywords: detrended fluctuation analysis (DFA), rank order statistics, heart rate variability (HRV), surgical intensive care units (SICU), electrocardiograph (ECG) signals

\section{INTRODUCTION}

Physiologic systems generate complex fluctuations in their output signals that reflect the

Received: Nov. 23, 2005; Accepted: Jan. 16, 2006

Correspondence: Jiann-Shing Shieh, Professor

Department of Mechanical Engineering, Yuan Ze University, 135 Yuan-Tung Rd., Chung-Li, Taoyuan, 320, Taiwan

E-mail: jsshieh@saturn.yzu.edu.tw underlying dynamics. Three particularly vexing features of physiologic time series are nonstationarity, nonlinearity and nonequilibrium phenomena. In the human body both the autonomic nervous system and the humoral system have direct effect on heart rate coordination [1-3]. Therefore, heart rate variability (HRV) is a powerful prognostic predictor of heart disease [4-9]. Finding and analyzing hidden dynamical structures of these signals are of basic and clinical interest. The traditional methods of analysis HRV 
situations time and frequency domain methods, are not enough. Recent studies have shown that the new nonlinear measures, particularly fractal analysis methods of heart rate dynamics, can detect subtle changes in heart rate behavior [10-15].

Human cardiac dynamics are driving by the complex nonlinear interactions of two competing forces: sympathetic stimulation increases heart rate, whereas parasympathetic stimulation decreases it. For this type of intrinsically noisy system, the novel technique of detrended fluctuation analysis (DFA) has been successfully developed to study nonstationary signals. DFA is a modified root-mean-square analysis of a random walk [16]. In recent years, the DFA method has become popular, and has been widely used to detect long-range or long-term (almost 24hr ECG recordings) correlation in nonstationary time series at various fields, such as the autonomic nervous system [3], ventricular fibrillation [4], congestive heart failure [10], dilated cardiomyopathy [11], protein representation [16-17], stock returns [18], electroencephalogram (EEG) in sleep [15,19-21], estimating dependence [22], periodic trends [23] and others. Although this asymptotic scaling exponent may serve as a useful index for selected diagnostic purpose, a one drawback is that very long data sets are required (at least 24 hours) for statistically robust results. For practical purposes, clinical investigators are usually interested in the possibility of using substantially shorter time series; but for very short time scales $(\mathrm{n}<10$ beats), the fluctuation is quite random. As the time scale becomes larger, the fluctuation becomes smoother [24]. Nevertheless, the short-term analysis of electrocardiograph (ECG) signals usually uses the time and frequency domain method [25-28]. Therefore, this study has two aims: (1) to study the short-term time series (i.e., $1 \mathrm{hr}$ heartbeat signals) of DFA in comparison with previous research (i.e., $24 \mathrm{hr}$ heartbeat signals), and (2) to investigate the short-term DFA method for analyzing heartbeat signals at SICU.

\section{THEORETICAL ANALYSES}

The DFA algorithm described elsewhere $[16,24,29]$, quantifies fractal-like correlation properties by calculating the scaling property of the root-meansquare fluctuation of the integrated and detrended time series data. For ECG signals analysis $24 \mathrm{hr}$ signals are always used. The DFA algorithm, and related twopoint correlation methods, such as Fourier transform and Hurst analyses, measure only one exponent characterizing a given signal. These methods, therefore, are most appropriate for the analysis of monofractal signals [29]. The theoretical relationship has been demonstrated between DFA and frequency- weighted power spectra [30-31]. To illustrate the DFA algorithm, we use the interbeat time series shown in Fig. 1(a) as an example. Briefly, the interbeat interval time series to be analyzed (with $\mathrm{N}$ samples) is first integrated as

$$
y(k)=\sum_{i=1}^{k}\left[B(i)-B_{\text {ave }}\right]
$$

where $B(i)$ is the ith interbeat interval and $B_{\text {ave }}$ is the average interbeat interval. Next, the integrated time series is divided into boxes of equal length, $n$. In each box of length $n$, a least-squares line is fit to the data (representing the trend in that box), as shown in Fig. 1(b). The y coordinate of the straight line segments is denoted by $y_{n}(k)$. Next, we detrend the integrated time series, $y(k)$, by subtracting the local trend, $y_{n}(k)$, in each box. The root-mean-square fluctuation of this integrated and detrended time series is calculated by

$$
F(n)=\sqrt{\frac{1}{N} \sum_{k=1}^{N}\left[y(k)-y_{n}(k)\right]^{2}}
$$

This computation is repeated over all time scales (box sizes) to characterize the relationship between $\mathrm{F}(\mathrm{n})$, the average fluctuation, as a function of box size. Typically, F(n) will increase with box size $n$. A linear relationship on a log-log plot indicates the presence of power law (fractal) scaling. Under such conditions, the fluctuations can be characterized by a scaling exponent $\alpha$, which is the slope of the line relating $\log F(n)$ to $\log n$.
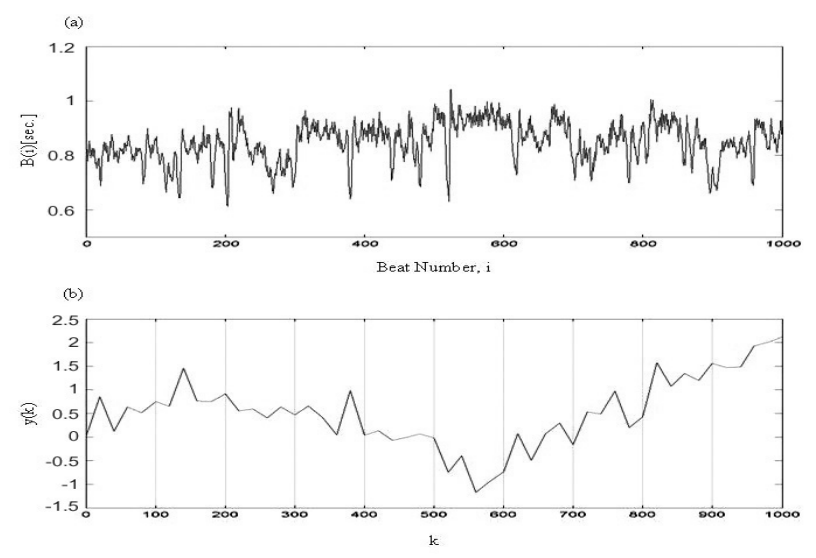

Fig 1. (a) Interbeat interval time series $B(i)$ of $\mathbf{1 0 0 0}$ beats. (b) The solid blue curve is the integrated time series: $y(k)=\sum_{i=1}^{k}\left[B(i)-B_{\text {ave }}\right]$, where $\boldsymbol{B}(\boldsymbol{i})$ is the $\mathbf{i}$-th interbeat interval shown in (a) and $B_{a v e}$ is the average interbeat interval. The vertical dotted lines indicate box of size $n=100$, the solid black straight lines segments are the estimated "trend" in each box by least-squares fit. 
In the DFA method, the fractal-like signal (1/f noise) results in exponent value $\alpha=1.0$, the white noise results in value 0.5 , and the Brownian noise in value 1.5. White noise indicates a simulated uncorrelated random time series. The white noise is the value at one instant that does not correlate with any previous value, and the Brownian noise is the integration of the white noise. The $1 / \mathrm{f}$ noise can be interpreted as a "compromise" between the complete unpredictability of white noise and the much smoother "landscape" of Brownian noise [3-4,10,13,19-21,29]. The phenomenon is that a larger value of the scale exponent $\alpha$ represents a smaller fractal dimension $[13,19-20,24,29]$.

\section{PARTICIPANT AND METHOD}

\subsection{Participant}

This study participants were divided into three groups as shown in Table I. From the ten college student volunteers aged 21-22 years in group A, ECG signals lasting around $1 \mathrm{hr}$ were collected. Ten white noise signals generated from a computer simulation as group B also provided ECG signals lasting around $1 \mathrm{hr}$. Finally, seventeen patients with 37 cases undergoing different types of neurosurgery for head injury and septicemia in the SICU of Tao-Yuan General Hospital, Tao-Yuan City, Taiwan were studied as group C. From this group aged 21-89 years, ECG signals lasting around $1 \mathrm{hr}$ were also collected. This study was approved by the local Ethics Committee and written informed consent was obtained from the next of kin of all patients.

\subsection{Method}

The volunteers ECG collection used an instrument operational amplifier, band-pass filter, level shifter, National Instrument AD card (NI card), and a notebook personal computer as shown in Fig. 2. In order to avoid losing ECG signals, the sampling frequency of data collection was set to $500 \mathrm{~Hz}$. The patients with head injury and septicemia of SICU were equipped with Hewlett Packard (HP) bedside monitors. The continuous ECG signals of these patients were

Table I. Patient and case number

\begin{tabular}{|c|c|c|}
\hline Groups & Patient number & Case number \\
\hline A healthy volunteers & 10 & 10 \\
\hline B white noise signals & 10 & 10 \\
\hline \multirow{2}{*}{ C SICU } & 15 & 25 \\
\hline & 2 & 12 \\
\hline
\end{tabular}

collected via NI card using a notebook personal computer as also shown in Fig. 2. After the ECG signals were collected on-line, the nonstationary signals were all obtained by off-line analysis. For this off-line analysis, we first collected the human heartbeat signals. Secondly, the peak of heartbeat signals to calculate the R-R interval time series was detected. Thirdly, the DFA algorithm was implemented to calculate the slope for the $\alpha$ values of different types of physiologic dynamics when changing the box of length $\mathrm{n}$ of interbeat interval time series. Finally, the slope value of $\log F(n)$ to $\log n$ plotted can be calculated by the DFA method. In addition, the investigator who designed this collecting system, was always present and responsible for handling any device or computer malfunction to bring the system back under control by the ICU surgeons.

\subsection{Statistical Analysis}

Values were expressed as means $\pm \mathrm{SD}$. Data were analyzed by one-way analysis of variance (ANOVA). The student-Newman-Keuls (SNK) test was conducted for multiple comparisons when the null hypothesis was not applicable. Differences were considered significant at a value of $\mathrm{P}<0.05$.

\section{RESULT}

From Table II, the mean values of the DFA scaling exponents $\alpha$ of groups $\mathrm{A}, \mathrm{B}$, and $\mathrm{C}$ are 0.958 $\pm 0.034,0.521 \pm 0.010$, and $0.815 \pm 0.183$, respectively. This is consistent with healthy human

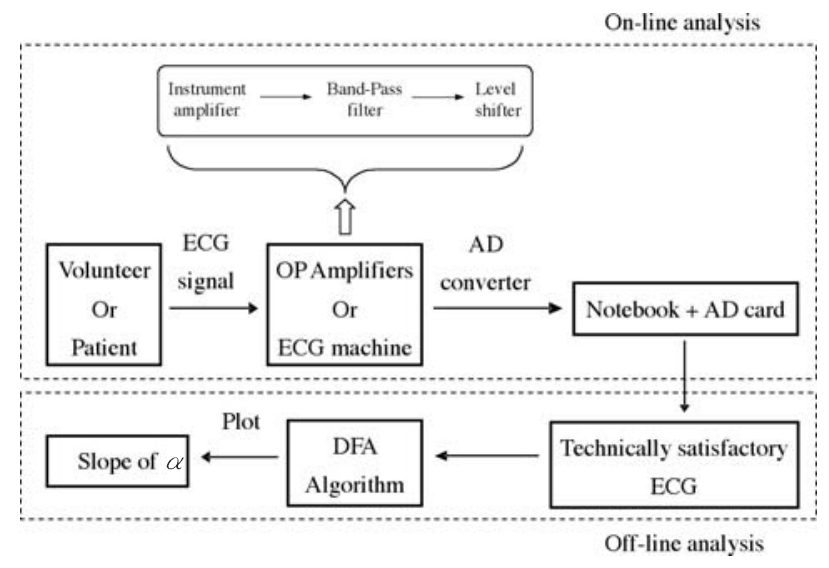

Fig 2. Block diagram for off-line analysis of slope of $\alpha$ and continuous on-line collection of volunteers ' ECG signals via an operational amplifier and National Instruments ' AD card using a notebook personal computer. 
Table $\Pi$. The $\alpha$ value and ANOVA statistical analysis of different groups of DFA method

\begin{tabular}{ccccc}
\hline Classification & Group A & Group B & Group C & P-Value \\
\hline$\alpha$ Value & $0.958 \pm 0.034^{a}$ & $0.521 \pm 0.010^{6}$ & $0.815 \pm 0.183^{c}$ & $<0.0001$ \\
\hline
\end{tabular}

$\alpha$ Value is expressed as mean $\pm \mathrm{SD}$ of different groups

$P<0.05$ was considered statistically significant using the ANOVA method

${ }^{a} P<0.05$ for Group A vs. Group B using the student-Newman-Keuls (SNK) test

${ }^{b} P<0.05$ for Group B vs. Group $\mathrm{C}$ using the student-Newman-Keuls (SNK) test

${ }^{c} P<0.05$ for Group C vs. Group A using the student-Newman-Keuls (SNK) test

value (i.e., $\alpha \approx 1.0$ ), and the white noise value (i.e., $\alpha$ $\approx 0.5$ ) from recent studies $[3,8,10,24,29]$. These results indicate similar results from short term data (i.e., $1 \mathrm{hr}$ ) compared with long term DFA computation results (i.e., $24 \mathrm{hr}$ ). Also, it was found that the $\alpha$ value of patients in the SICU was significantly lower $(\mathrm{P}<0.05)$ than healthy volunteers and significantly higher $(\mathrm{P}<$ $0.05)$ than white noise signals. Following to Yang et al. [12], the healthy volunteers (i.e., group A) and white noise signals (i.e., group B) were used as high and low limits of baseline for comparison with pathologic states in the cardiovascular intensive care units (CICU). Hence, it can be concluded that $\alpha$ values based on DFA statistics concept can clearly distinguish pathologic states in an SICU from the healthy group and the white noise signals. Finally, the typical examples of each case of full graphical complex physiologic fluctuations of cardiac interbeat interval time series of 2000 beats for a healthy volunteer, a patient with brain injury and septicemia in the SICU, and a white noise signal are shown in Fig. 3. Also, the plotted $\log _{10} F(n)$ vs. $\log _{10} n$ by the DFA analysis for each case of scaling exponents $\alpha$ is shown in Fig. 4 to demonstrate that $\alpha$ values based on DFA statistics from pathologic states in SICU are located between the healthy group and the white noise signals.

\section{CONCLUSION}

In this paper, the DFA method has been used to investigate the profiles of different types of physiologic dynamics. From experiment results, the $\alpha$ values for DFA obtained from healthy humans and from white noise were similar between $1 \mathrm{hr}$ and $24 \mathrm{hr}$ data. So, in the future it might not be necessary to collect and analyze the heartbeat data for 24 hours. In addition, the $\alpha$ index derived from the DFA method (a) Healthy volunteer

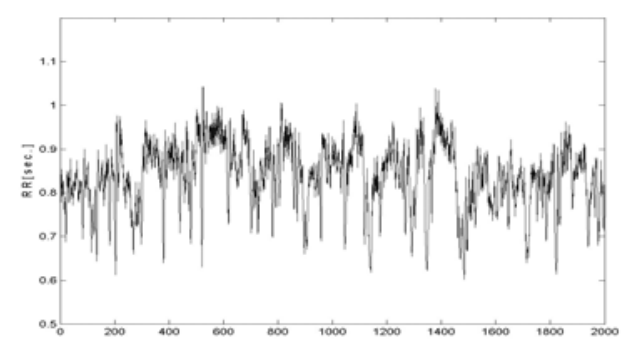

(b) Patient with brain injury

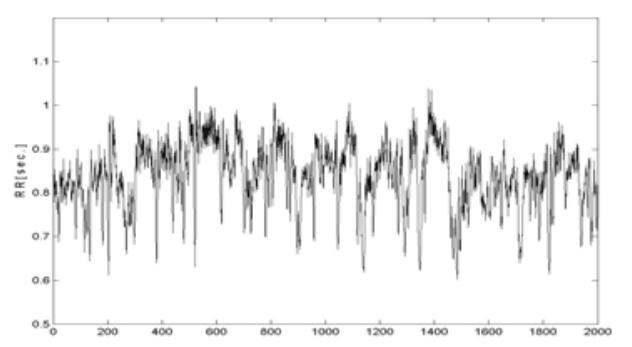

(c) Patient with septicemia

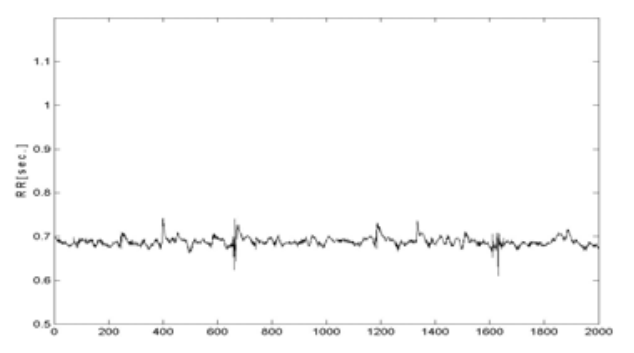

(d) White noise

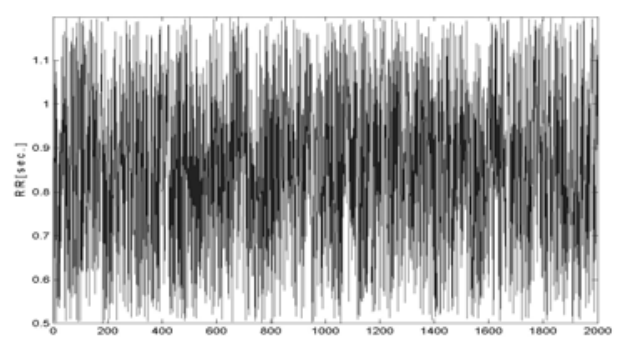

Fig 3. Representative complex physiologic fuctuations. Cardiac interbeat interval (normal sinus rhythm) time series of 2000 beats from (a) a healthy volunteer, (b) a patient with brain injury, (c) a patient with septicemia in the surgical intensive care unit, and (d) white noise. Note the highly nonstationary heart rate variations in (a), (b) and (c), such that the statistical properties change over relatively short time periods. Nonstationarity, as well as sustained oscillations, as seen in (d), suggest underlying nonlinear dynamics. 


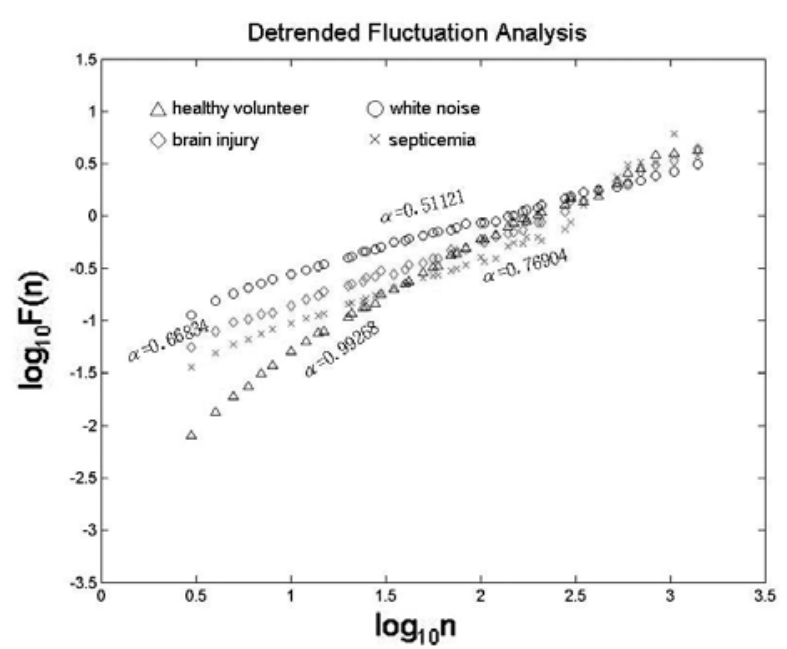

Fig 4. Fractal scaling analyses for four 1-hr interbeat interval time series. The triangles represent data from a healthy volunteer. The diamonds represent data from a patient with brain injury. The $\times s$ represent data from a patient with septicemia, whereas the circles are white noise time series. Plot of $\log _{10} F(n)$ vs. $\log _{10} n$ by the DFA analysis.

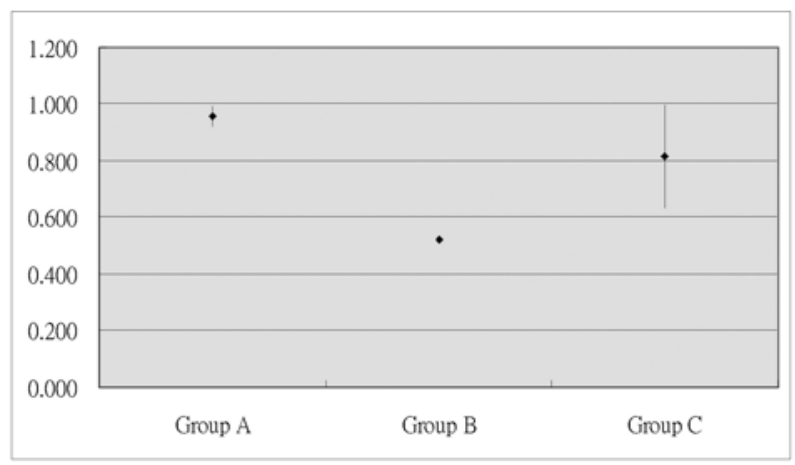

Fig 5. The $\alpha$ mean value of the interbeat interval time series derived from groups A, B, and C (Group A, healthy volunteers; Group B, white noise signals; Group C, surgical intensive care units ' patients). Values are given from the DFA method as mean \pm standard deviation.

was applied to patients in a SICU in comparison with healthy volunteers and white noise signals. The results have shown that this index can clearly distinguish pathologic states in the SICU from the healthy group and white noise signals. However, the variation of $\alpha$ value in Group $\mathrm{C}$ is still too large, as shown in Fig. 5.
In addition, the different clinical conditions are related to varying severity of the pathologic states. Hence, much research and greater numbers of patients are still necessary in order to differentiate the nonststionary indexes between different pathologic states (e.g., patients with brain injury or septicemia), and see how widely they are applicable. Furthermore, this method can be used for on-line monitoring of changes in patient status towards recovery or worsening condition in order to help surgeons diagnose patients in the SICU more rapidly in the future.

\section{ACKNOWLEDGEMENT}

The authors wish to thank the National Science Council (NSC) of Taiwan (Grant Number: NSC932213-E-155-051) for supporting this research.

\section{REFERENCE}

1. Akselrod S, Gordon D, Ubel FA, Shannon DC, Berger AC, Cohen RJ: Power spectral analysis of heart rate fluctuation: a quantitative probe of beat to beat cardiovascular control. Science 1981; 213: 220-222.

2. Hosaka H, Takase B, Katsushika S, Ohsuzu F, Kurita A: Altered fractal behavior and heart rate variability in daily life in neurally mediated syncope. Biomedicine \& Pharmacotherapy 2003; 57: $77 \mathrm{~s}-82 \mathrm{~s}$.

3. Nakamura T, Horio H, Miyashita S, Chiba Y, Sato $\mathrm{S}$ : Identification of development and autonomic nerve activity from heart rate variability in preterm infants. Bio Systems 2005; 79: 117-124.

4. M?kikallio TH, Koistinen J, Jordaens L, Tulppo MP, Wood N, Golosarsky B, Peng CK, Goldberger AL, and Huikuri HV: Heart rate dynamics before spontaneous onset of ventricular fibrillation in patients with healed myocardial infarcts. The American Journal of Cardiology 1999; vol. 83: 880-884.

5. Halberg F, Cornélissen G, Otsuka K, Schwartzkopff O, Halberg J, Bakken EE: Chronomics. Biomed Pharmacother 2001; 55: 153190.

6. Huikuri HV, M?kikallio TH: Heart rate variability in ischemic heart disease. Autonomic Neuroscience: Basic and Clinical 2001; 90: 95-101.

7. Reyners AKL, Hazenberg BPC, Reitsma WD and Smit AJ: Heart rate variability as a predictor of mortality in patients with AA and AL amyloidosis. European Heart Journal 2002; 23: 157-161.

8. Pagani M: Circadian heart rate and blood pressure 
variability considered for research and patient care. Int. J. Cardiol 2003; 87: 29-30.

9. Carpeggiani C, Abbate AL, Landi P, Michelassi C, Raciti M, Macerata A, Emdin M: Early assessment of heart rate variability is predictive of in-hospital death and major complications after acute myocardial infarction. International Journal of Cardiology 2004; 96: 361-368.

10. Stanley HE, Amaral LAN, Goldberger AL, Havlin S, Ivanov PCh, Peng CK: Statistical physics and physiology: monofractal and multifractal approaches. Physica A 1999; 270: 309-324.

11. Mahon NG, Hedman AE, Padula M, Gang Y, Savelieva I, Waktare JEP, Malik MM, Huikuri HV, McKenna WJ: Fractal correlation properties of R-R interval dynamics in asymptomatic relatives of patients with dilated cardiomyopathy. The European Journal of Heart Failure 2002; 4: 151158.

12. Yang ACC, Hseu SS, Yien HW, Goldberger AL, Peng CK: Linguistic analysis of the heartbeat using frequency and rank order statistics. Physical Review Letters 2003; v.90 (10): 108103-1-1081034.

13. Kubo Y, Murakami S, Matsuoka O, Hotta N, Oinuma S, Shinagawa M, Omori K, Nunoda S, Otsuka K, Ohkawa SI, Corn?lissen G, Halberg F: Toward chronocardiologic and chronomic insights: dynamics of heart rate associated with head-up tilting. Biomedicine \& Pharmacotherapy 2003; 57: 110s-115s.

14. Chiu HW, Wang TH, Huang LC, Tso HW, Kao T: The influence of mean heart rate on measures of heart rate variability as markers of autonomic function: a model study. Medical Engineering \& Physics 2003; 25: 475-481.

15. Staudacher M, Telser S, Amann A, Hinterhuber H, Ritsch-Marte M: A new method for change-point detection developed for on-line analysis of the heart beat variability during sleep. Physica A 2005; 349: 582-596.

16. Peng CK, Buldyrev SV, Havlin S, Simons M, Stanley HE, Goldberger AL: Mosaic organization of DNA nucleotides. Physical Review E 1994; 49: 1685-1689.

17. Yu ZG, Anh V, Lau KS: Fractal analysis ofmeasure representation of large proteins based on the detailed HP model. Physica A 2004; 337: 171-184.

18. Pilar GC: Long-range power-law correlations in stock returns. Physica A 2001; 299: 521-527.

19. Lee JM, Kim DJ, Kim IY, Park KS, Kim SI: Detrended fluctuation analysis of EEG in sleep apnea using MIT/BIH polysomnography data. Computers in Biology and Medicine 2002; 32: 3747.

20. Lee JM, Kim DJ, Kim IY, Park KS, Kim SI:
Nonlinear-analysis of human sleep EEG using detrended fluctuation analysis. Medical Engineering \& Physics 2004; 26: 773-776.

21. Stam CJ, Montez T, Jones BF, Rombouts SARB, van der Made Y, Pijnenburg YAL, Scheltens Ph: Disturbed fluctuations of resting state EEG synchronization in Alzheimer's disease. Clinical Neurophysiology 2005; 116: 708-715.

22. Weron R: Estimating long-range dependence: finite sample properties and confidence intervals. Physica A 2002; 312: 285-299.

23. Nagarajan R, Kavasseri RG: Minimizing the effect of periodic and quasi-periodic trends in detrended fluctuation analysis. Chaos, Solitons and Fractals 2005; 26: 777-784.

24. Peng CK, Havlin S, Stanley HE, Goldberger AL: Quantification of scaling exponents and crossover phenomena in nonstationary heartbeat time series. Chaos 1995; 5: 82-87.

25. Satoh K, Koh J, Kosaka Y: Effects of nitroglycerin on fractal features of short-term heart rate and blood pressure variability. Journal of Anesthesia 1999; 13: 71-76.

26. Migeotte PF and Verbandt Y: A novel algorithm for the heart rate variability analysis of short-term recordings: polar representation of respiratory sinus arrhythmia. Computers and Biomedical Research 1999; 32: 56-66.

27. Mäkikallio TH, Huikuri HV, Hintze U, Videbæk J, Mitrani RD, Castellanos A, Myerburg RJ, and Moller M, for the DIAMOND Study Group: Fractal analysis and time- and frequency-domain measures of heart rate variability as predictors of mortality in patients with heart failure. The American Journal of Cardiology 2001; Vol. 87: 178-181.

28. Elghozi JL, Girard A, Laude D: Effects of drugs on the autonomic control of short-term heart rate variability. Autonomic Neuroscience: Basic and Clinical 2001; 90: 116-121

29. Goldberger AL, Amaral LAN, Hausdorff JM, Ivanov PCh, Peng CK: Fractal dynamics in physiology: Alterations with disease and aging. Proceedings of the National Academy of Sciences of the United States of America 2002; vol.99 (suppl.1): 2466-2472.

30. Willson K, Francis DP, Wensel R, Coats AJS, Parker KH: Relationship between detrended fluctuation analysis and spectral analysis of heartrate variability. Physiological Measurement 2002; 23: $385-401$.

31. Willson K, Francis DP: A direct analytical demonstration of the essential equivalence of detrended fluctuation analysis and spectral analysis of RR interval variability. Physiological Measurement 2003; 24: N1-N7. 